The need for speed: how PD1-blockade only works if $\mathrm{T}$ cells are properly activated

\author{
Vom Berg, Johannes ; Kobold, Sebastian
}

\begin{abstract}
Programmed death receptor 1 (PD1) has been identified as critical negative regulator of T cell activity (1). While PD1-mediated T cell inhibition is an important mechanism to prevent autoimmunity, cancer and chronic infectious diseases may usurp this regulation mechanism to drive immune suppression (2). In fact, the PD1-PD-L1 axis is one of the major axis used by a variety of cancer types to inhibit $\mathrm{T}$ cell responses and function (3). As a direct consequence, strategies interfering with PD1-signaling such as antibodies have entered the clinic and are approved in a number of indications (4). A remarkable aspect being that these so-called check point inhibitors also show significant activity in such clinical situations where any other therapy had previously failed (5).
\end{abstract}

DOI: https://doi.org/10.21037/tcr.2017.06.55

Posted at the Zurich Open Repository and Archive, University of Zurich ZORA URL: https://doi.org/10.5167/uzh-143680

Journal Article

Published Version

Originally published at:

Vom Berg, Johannes; Kobold, Sebastian (2017). The need for speed: how PD1-blockade only works if T cells are properly activated. Translational Cancer Research, 6(S6):S1018-S1021.

DOI: https://doi.org/10.21037/tcr.2017.06.55 


\title{
The need for speed: how PD1-blockade only works if T cells are properly activated
}

\author{
Johannes vom Berg ${ }^{1}$, Sebastian Kobold ${ }^{2}$ \\ ${ }^{1}$ Institute of Laboratory Animal Science, University of Zurich, Zurich, Switzerland; ${ }^{2}$ Center of Integrated Protein Science Munich (CIPSM) and \\ Division of Clinical Pharmacology, Department of Medicine IV, Klinikum der Universitaet Muenchen, Munich 80337, Germany \\ Correspondence to: Sebastian Kobold, MD. Division of Clinical Pharmacology, Klinikum der Universitaet Muenchen, Lindwurmstraße 2a, Munich \\ 80337, Germany. Email: Sebastian.kobold@med.uni-muenchen.de. \\ Provenance: This is an invited Editorial commissioned by Section Editor Dr. Chen Qian (Center for Inflammation \& Epigenetics, Houston Methodist \\ Hospital Research Institute, Houston, TX, USA). \\ Comment on: Kamphorst AO, Wieland A, Nasti T, et al. Rescue of exhausted CD8 T cells by PD-1-targeted therapies is CD28-dependent. Science \\ 2017;355:1423-7.
}

Submitted Jun 19, 2017. Accepted for publication Jun 22, 2017.

doi: $10.21037 /$ tcr.2017.06.55

View this article at: http://dx.doi.org/10.21037/tcr.2017.06.55

Programmed death receptor 1 (PD1) has been identified as critical negative regulator of $\mathrm{T}$ cell activity (1). While PD1-mediated $T$ cell inhibition is an important mechanism to prevent autoimmunity, cancer and chronic infectious diseases may usurp this regulation mechanism to drive immune suppression (2). In fact, the PD1-PD-L1 axis is one of the major axis used by a variety of cancer types to inhibit $\mathrm{T}$ cell responses and function (3). As a direct consequence, strategies interfering with PD1-signaling such as antibodies have entered the clinic and are approved in a number of indications (4). A remarkable aspect being that these so-called check point inhibitors also show significant activity in such clinical situations where any other therapy had previously failed (5). PD1-blocking agents are under investigation in almost every cancer indication and the approved fields for therapy are constantly increasing (4). A major limitation is to differentiate patients who will benefit from this therapy from those who will not. Current strategies such as ligand expression in the tumor tissue, however, fail to reliably predict outcome, calling for additional research for patient stratification (6-8). Owing to the huge medical and economical impact disruption of the PD1-PD-L1 interactions has, a better understanding of PD1 biology and the identification of appropriate biomarkers emerge as a priority.

PD1 is part of a larger family of receptors and surface molecules: the CD28 superfamily of receptors (9). Early work on PD1 and other related receptors could demonstrate that their signaling motives are compatible and could be swapped to exchange the functions of the receptors $(10,11)$. CD28 can be turned into a suppressive molecule, if intracellular PD1 is fused to its C-terminus. Vice-versa, this compatibility can also be employed therapeutically, as PD1 can be fused to the CD28 signaling motives to generate a switch turning PD1 suppression into CD28 costimulation (12-14). Introducing such a PD1-CD28 switch in antigenspecific $\mathrm{T}$ cells can break suppression and enhance therapeutic $\mathrm{T}$ cell efficacy against tumors. It seems that this strategy can be employed both for TCR and chimeric antigen receptor transduced $\mathrm{T}$ cells. Such data demonstrates the value of simultaneous protection from PD1 inhibition and CD28 costimulation when the $\mathrm{T}$ cell meets its target.

A recent publication from Kamphorst and colleagues released in Science now demonstrated that CD28 on the $\mathrm{T}$ cell side and $\mathrm{B} 7$ on the tumor-bearing host side are necessary for full activity of PD1-blockade (15). Mechanistically, CD28 is required for adequate T cell proliferation upon PD1-antibody blockade and ultimately rescue of an exhausted phenotype and restoration of $\mathrm{T}$ cell effector function. The authors proved their hypothesis first in the LCMV chronic infection model and in the CT26 colon cancer model using different genetic deletion models for CD28 and blocking antibodies. In patients suffering from NSCLC receiving PD1-blockade in therapeutic intention, the authors found that $\mathrm{PD} 1^{+} \mathrm{T}$ cells responding through proliferation expressed CD28. They argue that 

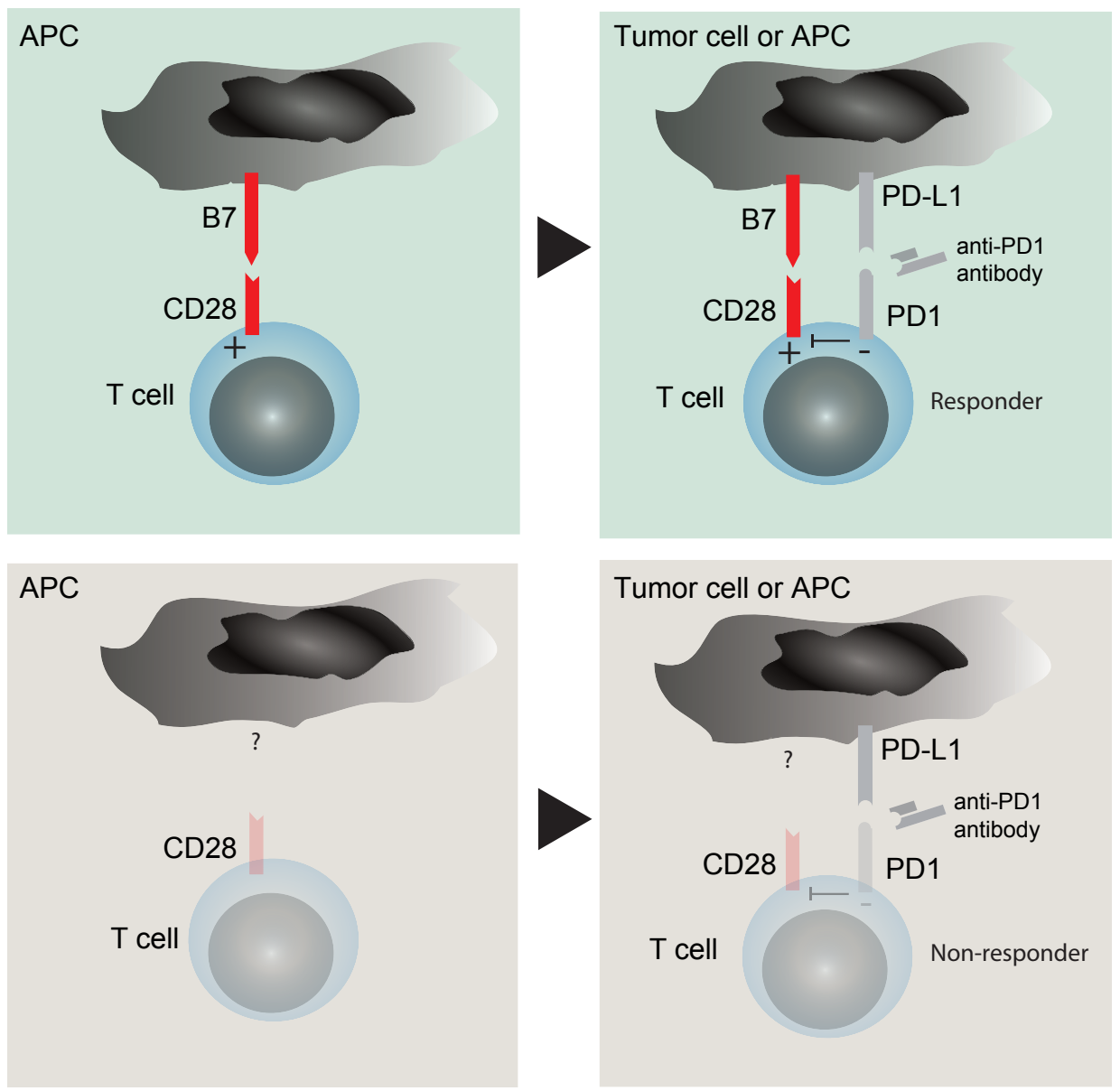

Figure 1 Prior activation is a pre-requisite for clinically relevant blockade of the PD1-PD-L1 axis. Recent studies by Kamphorst et al. and Hui et al. suggest that only after co-stimulation through CD28 engagement via B7 molecules on APCs, subsequent blockade of PD-1 (or its ligand PD-L1 on tumor cells or associated stroma cells or APCs) can lead to clinical benefit (light green, upper panels). In absence of costimulation, inert T cells do not react to PD1-PD-L1 pathway blockade (grey, lower panels). APC, antigen presenting cell; PD1, programmed death-1; PD-L1, PD1-ligand.

this finding corroborates the clinical relevance of their observations.

These results are remarkable on three levels (Figure 1): first, they imply that costimulation must be present for PD1-blockade activity. In other words, T cells must have already broken inertia and taken up speed through different layers of stimulation (signal one and two) for PD-1 to exert its full suppressive activity. It appears surprising and somehow counterintuitive as our text book knowledge argues that PD1-PD-L1 interactions happen at the effector site of a tumor or infected tissue where CD8+ T cells meet their antigen in the context of MHC and drive anergy (16). These novel insights now mean that costimulation through B7-molecules, probably coming from antigen presenting cells as main B7 expressors, must take place before $\mathrm{T}$ cell anergy can occur. Another possibility is simultaneous expression of B7 and PD-L1 on the tumor cell, which however, appears less likely as most cancer entities do not express costimulatory molecules. An unanswered question is whether costimulation and suppression must happen simultaneously and at the same site or if sequential binding would result in the same biological effect. Such consideration is important, as it would guide future work for the search of biomarkers and therapeutic applications.

Second, PD1 must somehow interfere with CD28 signaling or activity. Such consideration is unaddressed in the manuscript but would provide a more detailed mechanistical explanation to the observed findings. 
Again, our previous knowledge makes the point that downstream TCR signaling is the main target for PD1 dephosphorylation activity (17). This assumption is corroborated by a publication by Hui et al. who have conducted a detailed biochemical analysis of the targets for dephosphorylation activity of PD1(18). Remarkably, they found that unlike previously demonstrated the major targets are the tyrosine motives of CD28, providing direct evidence for the functional findings of Kamphorst et al. It would be of major interest to now understand the kinetics of the interactions: can dephosphorylation still occur if PD1 engagement was prior to CD28 activation? How long does the dephosphorylation last and can this effect be overrun by an excess of either signal? Such considerations might have a direct impact on the design of future therapies.

Third, the interplay between PD1 and CD28 might provide the long thought after reasoning on why PD-L1 expression does not predict response to PD1 blockade. The field has long been hoped for PD-L1 as a biomarker for PD1-blockade activity but controversial and inconsistent clinical data have proven its limitations (19). If the findings outlined by Kamphorst and colleagues hold true in a clinical setting, the requirement for $\mathrm{B} 7$ molecules might provide a direct explanation for the lack of predictive value of PDL1 expression. It then appears likely that either molecule might be limiting for the impact of PD1-mediated T cell inhibition. Thus, if $\mathrm{B} 7$ is not present PD1 might not be a major driver of $T$ cell anergy in a given patient and vice versa, if PD-L1 is not present or not where required, costimulation might not be the target of anergy and other mechanisms will kick in. However, several issues remain unaddressed: where do PD-L1 and B7 need to be expressed for PD1-mediated $\mathrm{T}$ cell suppression and to which degree? Is it sufficient if neighboring cells express either of them? Is simultaneous expression on the same cell necessary? These are questions that among others will need to be interrogated to further explore this clinical avenue.

In summary, the study by Kamphorst and coworkers have discovered a novel major mechanism used by PD1 to induce $\mathrm{T}$ cell anergy and required for PD1-blockade activity. Their study opens a new avenue of investigation if the mode of action of PD1 and on how this will be used to enhance the activity of immunotherapies and better predict their outcome.

\section{Acknowledgements}

Funding: S Kobold is supported by grants from the
Wilhelm Sander Stiftung (grant number 2014.018.1), the international doctoral program "i-Target: Immunotargeting of cancer" funded by the Elite Network of Bavaria, the Melanoma Research Alliance (grant number 409510), the Marie-Sklodowska-Curie "Training Network for the Immunotherapy of Cancer (IMMUTRAIN)" funded by the H2020 program of the European Union, the Else KrönerFresenius-Stiftung, the German Cancer Aid, the ErnstJung-Stiftung, by LMU Munich's Institutional Strategy LMUexcellent within the framework of the German Excellence Initiative and the Bundesministerium für Bildung und Forschung. J vom Berg is supported by grants of the University of Zurich (FK-15-057), the Novartis Foundation for medical-biological Research (16C231) and Swiss Cancer Research (KFS-3852-02-2016).

\section{Footnote}

Conflicts of Interest: The authors have no conflicts of interest to declare.

\section{References}

1. Latchman Y, Wood CR, Chernova T, et al. PD-L2 is a second ligand for PD-1 and inhibits T cell activation. Nat Immunol 2001;2:261-8.

2. Mellman I, Coukos G, Dranoff G. Cancer immunotherapy comes of age. Nature 2011;480:480-9.

3. Luke JJ, Flaherty KT, Ribas A, et al. Targeted agents and immunotherapies: optimizing outcomes in melanoma. Nat Rev Clin Oncol 2017. [Epub ahead of print].

4. Kobold S, Duewell P, Schnurr M, et al. Immunotherapy in Tumors. Dtsch Arztebl Int 2015;112:809-15.

5. Ansell SM, Lesokhin AM, Borrello I, et al. PD-1 Blockade with Nivolumab in Relapsed or Refractory Hodgkin's Lymphoma. N Engl J Med 2015;372:311-9.

6. Topalian SL, Hodi FS, Brahmer JR, et al. Safety, activity, and immune correlates of anti-PD-1 antibody in cancer. $\mathrm{N}$ Engl J Med 2012;366:2443-54.

7. Reck M, Rodriguez-Abreu D, Robinson AG, et al. Pembrolizumab versus Chemotherapy for PD-L1Positive Non-Small-Cell Lung Cancer. N Engl J Med 2016;375:1823-33.

8. Borghaei H, Paz-Ares L, Horn L, et al. Nivolumab versus Docetaxel in Advanced Nonsquamous Non-Small-Cell Lung Cancer. N Engl J Med 2015;373:1627-39.

9. Sharpe AH, Freeman GJ. The B7-CD28 superfamily. Nat Rev Immunol 2002;2:116-26. 
10. Chemnitz JM, Parry RV, Nichols KE, et al. SHP-1 and SHP-2 associate with immunoreceptor tyrosine-based switch motif of programmed death 1 upon primary human $\mathrm{T}$ cell stimulation, but only receptor ligation prevents $\mathrm{T}$ cell activation. J Immunol 2004;173:945-54.

11. Riley JL, June CH. The CD28 family: a T-cell rheostat for therapeutic control of T-cell activation. Blood 2005;105:13-21.

12. Kobold S, Grassmann S, Chaloupka M, et al. Impact of a New Fusion Receptor on PD-1-Mediated Immunosuppression in Adoptive T Cell Therapy. J Natl Cancer Inst 2015;107(8). pii: djv146.

13. Ankri C, Shamalov K, Horovitz-Fried M, et al. Human $\mathrm{T}$ cells engineered to express a programmed death $1 / 28$ costimulatory retargeting molecule display enhanced antitumor activity. J Immunol 2013;191:4121-9.

14. Liu X, Ranganathan R, Jiang S, et al. A Chimeric SwitchReceptor Targeting PD1 Augments the Efficacy of

Cite this article as: vom Berg J, Kobold S. The need for speed: how PD1-blockade only works if $\mathrm{T}$ cells are properly activated. Transl Cancer Res 2017;6(Suppl 6):S1018-S1021. doi: 10.21037/tcr.2017.06.55
Second-Generation CAR T Cells in Advanced Solid Tumors. Cancer Res 2016;76:1578-90.

15. Kamphorst AO, Wieland A, Nasti T, et al. Rescue of exhausted CD8 T cells by PD-1-targeted therapies is CD28-dependent. Science 2017;355:1423-7.

16. Topalian SL, Taube JM, Anders RA, et al. Mechanismdriven biomarkers to guide immune checkpoint blockade in cancer therapy. Nat Rev Cancer 2016;16:275-87.

17. Zinselmeyer BH, Heydari S, Sacristan C, et al. PD-1 promotes immune exhaustion by inducing antiviral $\mathrm{T}$ cell motility paralysis. J Exp Med 2013;210:757-74.

18. Hui E, Cheung J, Zhu J, et al. T cell costimulatory receptor $\mathrm{CD} 28$ is a primary target for $\mathrm{PD}-1-$ mediated inhibition. Science 2017;355:1428-33.

19. Fusi A, Festino L, Botti G, et al. PD-L1 expression as a potential predictive biomarker. Lancet Oncol 2015;16:1285-7. 